\title{
Transient Surface Impedance Measurements using Spherics - Source Identification and Characterisation
}

\author{
David V. Thiel ${ }^{1} \quad$ Gavin Mogensen ${ }^{1}$
}

\begin{abstract}
Transient recordings of the electric and magnetic fields over the audio-band display significant transient pulses (spherics) which can be used for surface impedance measurements providing the lighting strike is distant and predominantly vertical. Horizontal lighting strikes, other atmospheric noise and instrument noise creates incorrect surface impedance data. Automated spheric selection can be made by calculating the sliding window correlation coefficient, and observing a period of elevated values followed by a rapid transition to a negative value. Data selected in this manner yield coherent surface impedance values.
\end{abstract}

\section{INTRODUCTION}

Single frequency surface impedance measurements are used in subsurface probing of the earth to depths of up to 100 metres [1]. These measurements rely on VLF transmissions from navigation beacons located around the world. The signals are vertically polarised and provide almost world-wide coverage.

Spherics are electromagnetic signals generated by distant (i.e. greater than $500 \mathrm{~km}$ ) lightning strikes. While at the source, the strike duration is less than tens of microseconds, atmospheric dispersion along the propagation path typically results in a $3 \mathrm{~ms}$ signature, often with a low frequency "tail" (see Figure 1). Recordings of the electric and magnetic fields of spherics (lightning generated radio signals) can be used for surface impedance measurements [2]. These powerful radio pulses can be up to 10 to 100 times greater than the noise level, and usually contain more than five zero-crossings with decreasing frequency. Unfortunately impulsive recordings can result from nearby noise sources and non-vertical lightning stokes. Rather than acquire and analyse all data, discrimination of the signals before processing can significantly reduce the computational power required to gain useful surface impedance information.

Observations of spherics from distant lightning strikes in the ELF-VLF spectral bands occur at rates of more than one every second [3], and major cloud to ground lightning strikes which generate sufficient energy to travel around the world have been observed at more than 2 per minute [4].

A randomly polarised radio signal reflected from a lossy, planar surface can be analysed using Fresnel reflection coefficients of the parallel and perpendicular field components. From this, the surface impedance can be determined. For clarity in the analysis, it is useful to use the signals from predominantly vertically oriented lighting strikes and discard others. There is clearly a need to distinguish between these two cases. If the earth has two or three dimensional structures, the surface impedance is dependent on the plane of incidence of the radiation. In magnetotelluric investigations, crossed dipole and loop antennas are used to record the two dimensional surface impedance [5]. For reasons of field survey efficiency, only one dimensional measurements are used, and so only one dimensional recordings are considered in this paper. The variation of surface impedance with frequency is limited theoretically, and so rapid variations in the surface impedance spectra are not physically possible.

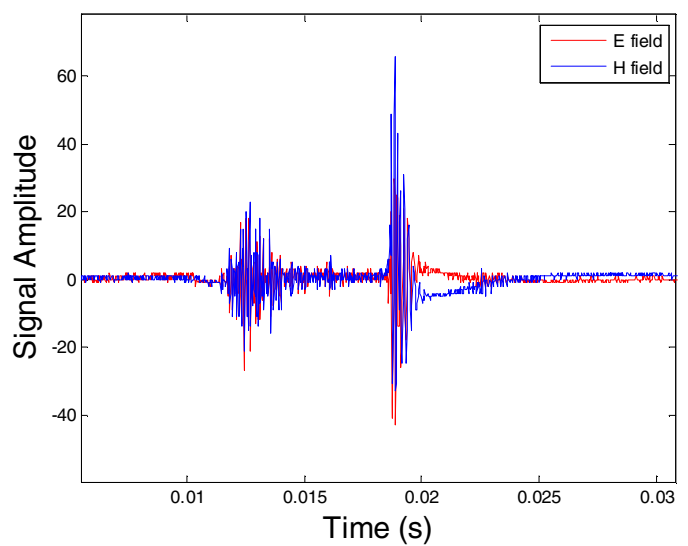

Figure 1: Typical E and $\mathrm{H}$ field recordings of two spherics.

\section{SPHERIC RECORDINGS}

Time series data was acquired at a remote field site in Queensland where mains frequency $(50 \mathrm{~Hz})$ and local electromagnetic noise levels were low. The sampling rate was $50 \mathrm{kS} / \mathrm{s}$ using a 12 bit ADC. The electric field antenna was a balanced, insulated dipole lying on the earth and the $\mathrm{H}$ field antenna was a multi-turn, coil with a ferrite core. The dipole was oriented north-south and the axis of the coil was oriented eastwest. This provides maximum response to signals arising from the thunderstorm cells located near the equator. The response of an electrically short dipole

\footnotetext{
${ }^{1}$ Centre for Wireless Monitoring and Applications, Griffith University, Nathan, Qld 4111, Australia, e-mail: d.thiel@griffith.edu.au, tel.: +617 37357192, fax: +617 37355198.
} 
is proportional to $l / \lambda$ (the length of the dipole $l$ in wavelengths $\lambda$ ), and so is inversely proportional to the frequency. The response of an electrically small loop antenna is proportional to $A / \lambda^{2}$ (the area $A$ in wavelengths squared) and so is inversely proportional to the frequency squared.

The ambient noise level was \pm one bit for both the electric and magnetic fields. Recordings were made for several minutes at the same location. Magnetic field deviations of greater than \pm 25 bits were excised from the record in $4 \mathrm{k}$ sample records for later analysis.

\section{SURFACE IMPEDANCE THEORY}

Radio signals in the band $500 \mathrm{~Hz}-20 \mathrm{kHz}$ propagate long distances in the earth-ionosphere waveguide [6]. A vertically polarized signal incident on the earth's surface is partly reflected and partly penetrates the surface. The surface impedance $Z_{s}$ at angular frequency $\omega$, is defined as [5]

$$
Z_{s}(\omega)=\frac{E_{x}(\omega)}{H_{y}(\omega)}
$$

where $E_{x}$ is the horizontal electric field measured on the surface of the earth and $H_{y}$ is the horizontal magnetic field measured perpendicular to the electric field component. The magnetic field is the primary field and the energy source and the horizontal electric field is the secondary reradiation from the earth. Thus a spheric with no energy at a particular frequency will result in the surface impedance being undefined.

A time domain recording of the electric and magnetic fields must be converted to the frequency domain to calculate the surface impedance. This data can be returned to the time domain to determine the surface impedance as a function of time [7].

For an infinitely deep, conducting earth plane, the surface impedance is approximately proportional to the square root of the frequency [6]. The lower frequencies have an increased depth of penetration (skin depth) and so an increased probing depth. While higher frequencies have less penetration, they have improved depth resolution. For this reason, surface impedance measurements across a wide frequency range is highly desirable for earth probing.

\section{TRANSIENT DATA ANALYSIS}

In order to rapidly assess the quality of the incoming data stream, the evaluation must be undertaken on the time series. Figure 2 (top trace) shows a typical time series recorded at the field site. Three significant events can be observed $(t=0.013 \mathrm{~s}, 0.019 \mathrm{~s}$ and $0.069 \mathrm{~s})$. A measure of energy content in an $\mathrm{AC}$ coupled signal is the root mean squared (RMS) value.
The electric field RMS value, $E_{R M S}$ can be calculated using the equation

$$
E_{R M S}=\frac{\sqrt{n \sum_{i=1}^{n} E_{i}^{2}-\left(\sum_{i=1}^{n} E_{i}\right)^{2}}}{n}
$$

where $n$ is the number of samples in the window and $E_{i}$ is the $i^{\text {th }}$ field value. Figure 2 (middle trace) was calculated using a sliding $n=60$ window. This is equivalent to $1.5 \mathrm{~ms}$ of one half of the minimum time for a useful spheric. There is clearly significant energy in three spherics noted previously. The signal to noise ratio is greater than 10:1 for these three events. The electric field also shows significant energy at these times although this is dependent on the ground conductivity. Of significance, however, is the difference in the ratio $E_{R M S} / H_{R M S}$. The three events have ratios of $0.96,0.77$ and 0.83 respectively. Other, less significant events are observed in the record, but the signal to noise ratio is less than $3: 1$.

The correlation coefficient $R^{2}$ between two signals recorded over the same sliding window $(n=60)$ at the same time can be calculated using the equation:

$$
R^{2}=\frac{n \sum_{i=1}^{n} E_{i}-\sum_{i=1}^{n} E_{i} H_{i}}{E_{R M S} H_{R M S}}
$$

where $H_{R M S}$ is calculated using a formula for magnetic field similar to equation (2).
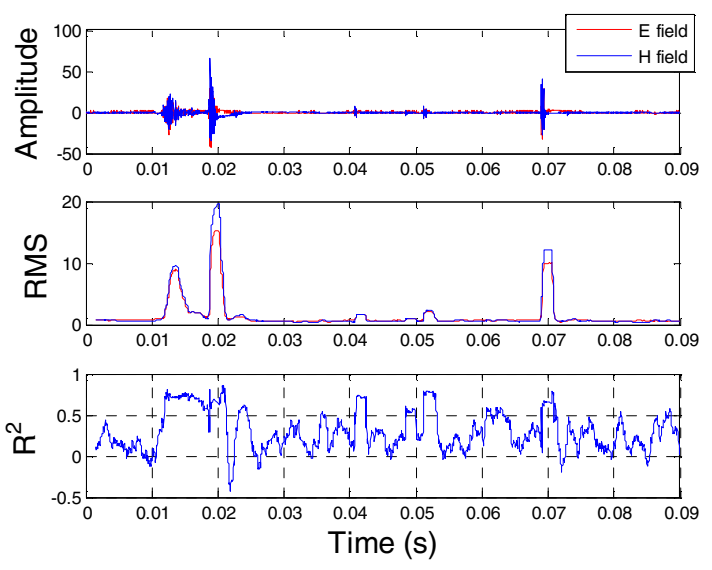

Figure 2: A spheric recording (top) and the corresponding sliding window RMS field strength (centre) and sliding window correlation coefficient

(bottom). The window length was $1.5 \mathrm{~ms}$.

The correlation coefficient $R^{2}$ is plotted in Figure 2 (bottom trace). It is clear that all three significant events and some other minor events result in a significant and stable value of $R^{2}$ over the length of the window. 
A further examination of the $R^{2}$ plot, reveals that the second and last significant spherics have a characteristic profile with a period of constant, elevated values, followed by a positive spike immediately followed by a significant negative spike. A significant negative value of $R^{2}$ indicates that the two signals are 180 degrees out of phase. This corresponds to the low frequency tail evident on the raw field time series data. This tail is not evident on the first significant event.

\section{SURFACE IMPEDANCE ANALYSIS}

The time series data was converted to the frequency domain using the Fourier transformation. The data was then corrected for the frequency dependence of the antennas, and used the surface impedance was calculated using equation 1 . This is only valid when there is significant energy in the magnetic field spectrum. The three spherics shown in figure 2 were used individually to calculate the surface impedance. The $\mathrm{E}$ and $\mathrm{H}$ field spectra and the magnitude $\left|Z_{s}\right|$, and phase $\operatorname{Arg}\left(Z_{s}\right)$, of the surface impedance are shown in Figures 3, 4 and 5 respectively. In order to reduce the noise while preserving the frequency resolution, all data was set to zero apart from the period of the spheric. As the spherics were recorded at the same location, an analysis of the surface impedance should yield similar values providing the SNR is sufficient. $\left|Z_{S}\right|$ values are relative values as the antennas were not calibrated.
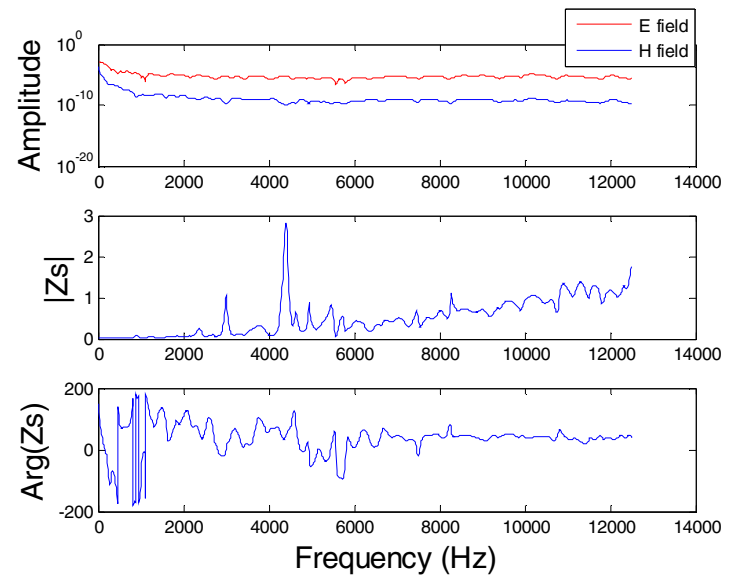

Figure 3: Surface impedance data from spheric 1.

\subsection{Spheric \#1}

Despite the relatively high energy in this spheric (Figure 3), the surface impedance profile (both amplitude and phase) appears noisy indicating that the origin of the noise pulse is not likely to be a distant vertically polarized lightning source. In addition the field spectra do not show characteristic variations associated with ionospheric band pass characteristics. The frequency trend of the surface impedance however is consistent with the other two recordings.
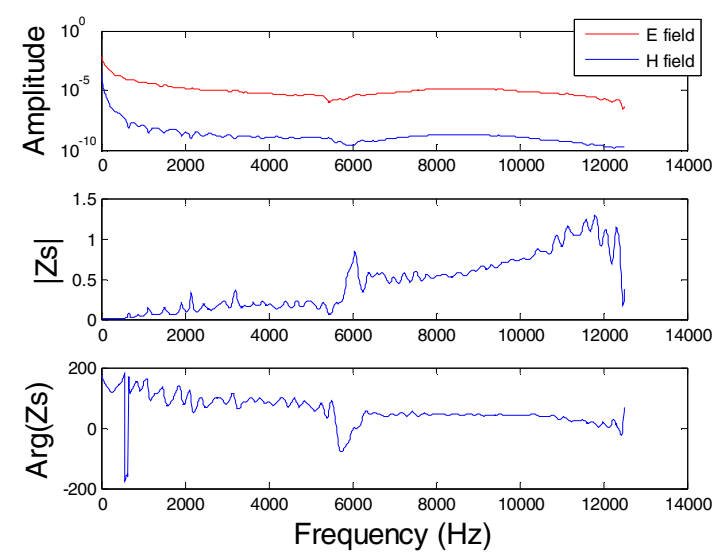

Figure 4: Surface impedance data from spheric 2.

\subsection{Spheric \#2}

This spheric spectrum has a notch in the field strength at approximately $6 \mathrm{kHz}$ in both the electric and magnetic fields. This is related to the cut-off frequency for the earth-ionosphere wave guide [8]. The surface impedance values calculated from such low magnetic signal levels deviate significantly from adjacent measurements and can not be interpreted in terms of the earth's subsurface structure. Compared to spheric 1, the surface impedance data shows less noise and the phase is consistent in the band $7 \mathrm{kHz}$ to $11 \mathrm{kHz}$. This is more likely to be a vertically polarized lightning strike.
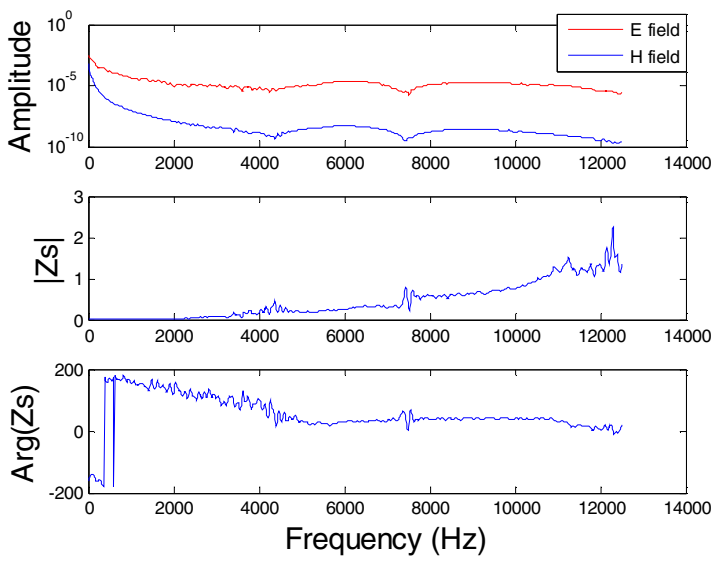

Figure 5: Surface impedance data from spheric 3.

\subsection{Spheric \#3}

The spectra from this record has notch band characteristics at both $4.4 \mathrm{kHz}$ and $7.3 \mathrm{kHz}$. The surface impedance amplitude and phase record appear relatively noise free and the phase is quite consistent over the frequency range $5-7 \mathrm{kHz}$ and 7.9 
$11 \mathrm{kHz}$. This is likely to be a vertically polarized, distant spheric.

\section{DISCUSSION AND CONCLUSIONS}

The time domain analysis of streaming electric and magnetic field data has been examined for efficacy in determining broad band surface impedance. While sliding window RMS field strength calculations of the magnetic field can be used to determine the signal to noise ratio, some noises bursts can exceed a preset threshold suitable for spheric data acquisition. The inclusion of these noise bursts in the surface impedance determination using mean values will result in significant error.

The $E_{R M S} / H_{R M S}$ ratio for the three spherics had significantly different values. This is not unexpected as both the surface impedance and the antennas are frequency dependent. Spheric 1 with the highest RMS ratio does not have a significant low frequency tail, whereas a higher proportion of the energy in spheric 2 is found in this tail.

The application of a sliding window correlation coefficient was not sufficiently selective based on values alone. However, a characteristic signature was observed which can identify useful data. This signature requires a prolonged elevated $R^{2}$ value followed by a rapid transition to a negative value. The negative value corresponds to the low frequency tail observed in the raw data where the $\mathrm{E}$ and $\mathrm{H}$ fields are 180 degrees out of phase.

In this paper, a comparison between three recorded noise bursts is reported. This technique was subsequently applied to a number of data sets with useful coherent surface impedance data being obtained. The main reason for this investigation was an exploration of a selection technique what can be applied to in-field, real time data acquisition and analysis. Further work will explore the method over a range of earth structures. In particular, the success of the technique on field measurements made in the vicinity of two dimensional and three dimensional earth materials must be assessed before it can be used routinely.

\section{Acknowledgments}

The authors acknowledge support from an Australian Postgraduate Scholarship. Data and discussions with Yvette O'Keefe and Steven O'Keefe are greatly appreciated.

\section{References}

[1] D.V. Thiel, "Relative wave tilt measurements at VLF." Geoexploration 17 (4), pp. 285-292, 1979.

[2] D.V. Thiel, M.J. Wilson, and C.J. Webb, "A surface impedance mapping technique based on radiation from discrete lightning strokes." Geoexploration 25 pp. 163-172, 1988.

[3] World wide lighting location network (WLLN) http://webflash.ess.washington.edu/spectra.html accessed 30 May 2010.

[4] T. Ogawa, and M. Komatsu, "Propagation velocity of VLF-EM waves from lightning discharges producting Q-bursts observed in the range 10-15 Mm", Atmosph. Res. 95, pp. 101-107, 2010.

[5] F.E.M. Lilley, "Magnetotelluric tensor decomposition: Part I, Theory for a basic procedure", Geophysics, 63 (6), pp. 1885-1897, 1997.

[6] J.R. Wait, Electromagnetic waves in stratified media, Pergamon Press, NY, 1962

[7] Y. O’Keefe, S.G. O'Keefe, and D.V. Thiel, "Time dependent surface impedance from spherics", IEEE Geoscience and Remote Sensing Letters, 2 (2), pp. 104-107, 2005.

[8] J. Galejs, Terrestrial propagation of long electromagnetic waves, Pergamon Pres, NY, 1972. 